\title{
AN EXISTENCE THEOREM FOR A MODIFIED SPACE-INHOMOGENEOUS, NONLINEAR BOLTZMANN EQUATION
}

\section{BY LEIF ARKERYD}

Communicated by Felix Browder, December 29, 1971

1. Preliminaries. Consider the initial value problem for the following class of nonlinear Boltzmann equations:

$$
\frac{\partial}{\partial t} f+v \nabla_{x} f=Q f \quad(t>0), f(0)=f_{0} \geqq 0,
$$

with collision operator

$$
Q f\left(x, v_{1}\right)=\int_{\mathbf{R}^{3} \times \mathbf{B}^{2}}\left[J_{u}^{*}\left\langle f_{1} f_{2}\right\rangle-\left\langle f_{1} f_{2}\right\rangle\right] k d u d v_{2} .
$$

The space coordinate $x$ belongs to a parallelepiped

$$
\omega=\left\{x=\left(x_{1}, x_{2}, x_{3}\right) ;\left|x_{j}\right| \leqq a_{j} / 2\right\},
$$

and

$$
\begin{aligned}
\left\langle f_{1} f_{2}\right\rangle & =f\left(x, v_{1}\right) f\left(x, v_{2}\right) & & \text { if }\left|f\left(x, v_{1}\right) f\left(x, v_{2}\right)\right| \leqq N, \\
& =N \operatorname{sign} f\left(x, v_{1}\right) f\left(x, v_{2}\right) & & \text { otherwise. }
\end{aligned}
$$

The impact parameter $u$ in $R^{2}$ is, for convenience, restricted to the disc

$$
B^{2}=\left\{u \in R^{2} ;|u| \leqq 1 / \sqrt{\pi}\right\} .
$$

The kernel $k\left(v_{1}, v_{2}\right)$ is a measurable, nonnegative, and bounded function vanishing for $\left|v_{1}\right|^{2}+\left|v_{2}\right|^{2}>K_{1}^{2}$, with $k\left(v_{1}, v_{2}\right)=k\left(v_{2}, v_{1}\right)$ and invariant under $J^{*}, J^{*} k=k$. Here $J^{*}$ is induced by a $C^{1}$-diffeomorphism $J$ on $R^{3} \times R^{3} \times B^{2}$, restricted in a certain way. With the velocity mappings

$$
\begin{aligned}
& \text { 1: } R^{3} \times R^{3} \rightarrow R:\left(v_{1}, v_{2}\right) \rightarrow 1, \\
& p: R^{3} \times R^{3} \rightarrow R^{3}:\left(v_{1}, v_{2}\right) \rightarrow v_{1}+v_{2}, \\
& T: R^{3} \times R^{3} \rightarrow R:\left(v_{1}, v_{2}\right) \rightarrow\left|v_{1}\right|^{2}+\left|v_{2}\right|^{2}, \\
& \Sigma: R^{3} \times R^{3} \rightarrow R^{3} \times R^{3}:\left(v_{1}, v_{2}\right) \rightarrow\left(v_{2}, v_{1}\right),
\end{aligned}
$$

the restriction on the (collision) mapping $J$ can be written

$$
1 \circ J_{u}=1, \quad p \circ J_{u}=p, \quad T \circ J_{u}=T,
$$

AMS 1969 subject classifications. Primary 8245, 8220.

Copyright (C) American Mathematical Society 1972 


$$
\begin{aligned}
\Sigma \circ J_{u} & =J_{u} \circ \Sigma, \\
J \circ J & =\text { identity } .
\end{aligned}
$$

For a discussion of the impact parameter $u$, the kernel $k$ and the collision mapping $J$, see [1].

We now consider $Q$. As usual $Q$ is zero for the Maxwell distributions, which thus are equilibrium solutions. A basic assumption in the deduction of the Boltzmann equation in kinetic gas theory is the low density of the gas. Then the contribution of the higher collisions are neglected in the development of the gas on physical grounds, so that the binary collisions alone are responsible for the development of the gas through the binary collision operator. For the same physical reasons an additional term should be inserted in case high densities develop in the gas. In the present paper that additional term cancels the binary collisions at high densities. But the proofs below hold for more general additional terms, thus under more general assumptions on the behaviour of the gas at high densities.

Boltzmann used $k=C\left|v_{1}-v_{2}\right|$ as the weight-function in $Q$. In the present paper, however, we use for the physical case

$$
\begin{aligned}
k & =C\left|v_{1}-v_{2}\right| & & \left(\left|v_{1}\right|^{2}+\left|v_{2}\right|^{2} \leqq K_{1}^{2}\right), \\
& =0 & & \left(\left|v_{1}\right|^{2}+\left|v_{2}\right|^{2}>K_{1}^{2}\right) .
\end{aligned}
$$

We consider (1.1) written as

$$
\frac{d}{d t} f\left(x+v_{1} t, v_{1}, t\right)=Q f\left(x+v_{1} t, v_{1}, t\right) \quad(t>0), f(0)=f_{0} \geqq 0,
$$

$f$ here denoting the periodic continuation in $x$ with period $\omega$ of the function $f$ in (1.1). The main result is an existence and uniqueness theorem for (1.6), when $f_{0} \in L^{\infty}\left(\omega \times R^{3}\right)$. We also prove the moment conservation laws and the usual laws of macroscopic gas dynamics as well as the $\mathscr{H}$-theorem.

As in the bounded space-homogeneous case treated in [1], the existence and uniqueness is based on the local Lipschitz continuity of $Q$ and the positivity of a related operator. The method also works in more general situations, e.g. for a containor $\omega$ with sufficiently well-behaved boundary under specular reflexion and a constant exterior force.

2. Results. We notice that $Q f\left(x+v_{1} t, v_{1}, t\right)$ does not depend on $\left|v_{1}\right|>K_{1}$. Hence it is enough to consider initial data $f_{0} \in L^{\infty}(\Omega)$ with

$$
\Omega=\left\{(x, v) ; x \in \omega,|v| \leqq K_{1}\right\},
$$

and to consider the solution $f$ for $t>0$ as a function of $v$ for $|v| \leqq K_{1}$ and periodic in $x$ with period $\omega$.

We also notice that 


$$
\begin{aligned}
\left|\left\langle f_{1} f_{2}\right\rangle-\left\langle g_{1} g_{2}\right\rangle\right| \leqq & \left|f\left(x, v_{1}\right)\right|\left|f\left(x, v_{2}\right)-g\left(x, v_{2}\right)\right| \\
& +\left|g\left(x, v_{2}\right)\right|\left|f\left(x, v_{1}\right)-g\left(x, v_{1}\right)\right| .
\end{aligned}
$$

Then $Q$ is locally Lipschitz continuous,

$$
\|Q f-Q g\|_{\infty} \leqq K_{2}\left(\|f\|_{\infty}+\|g\|_{\infty}\right)\|f-g\|_{\infty},
$$

if \|\|$_{\infty}$ denotes the norm in $L^{\infty}(\Omega)$ and $K_{2}=2 \sup k 3^{-1} 4 K_{1}^{3} \pi$.

THEOREM 2.1. There exists a unique, nonnegative solution $f(x, v, t)$ $\in L^{\infty}(\Omega)(t>0)$ of $(1.6)$ for every $f_{0} \geqq 0$ in $L^{\infty}(\Omega)$.

Proof. The local existence follows as in the space-homogeneous case of [1]. We repeat it for the convenience of the reader. By (2.1), $Q$ is locally Lipschitz continuous, and so there exists a unique solution $f$ of (1.6) for $0 \leqq t \leqq t_{0}, t_{0}>0$ only depending on $K_{2}$ and $\left\|f_{0}\right\|_{\infty}$. But $\|Q(f, f)\|_{\infty}$ $\leqq N K_{2}$ and

$$
\begin{gathered}
\|f(t)\|_{\infty} \leqq\left\|f_{0}\right\|_{\infty}+\int_{0}^{t} N K_{2}=\left\|f_{0}\right\|_{\infty}+t N K_{2}, \\
\sup _{\Omega}\left|f\left(x+v t_{1}, v, t_{1}\right)-f\left(x+v t_{2}, v, t_{2}\right)\right| \leqq N K_{2}\left|t_{1}-t_{2}\right| .
\end{gathered}
$$

We conclude by the usual continuation argument that $f$ exists for all $t>0$.

The proof is complete if we can show the positivity of $f$. Consider the equation

$$
\begin{array}{r}
g\left(x+v_{1} t, v_{1}, t\right)=\exp \left[-H\left(x+v_{1} t, t\right)\right] \cdot f_{0}\left(x, v_{1}\right) \\
+\int_{0}^{t} \exp \left[-H\left(x+v_{1} t, t\right)+H\left(x+v_{1} s, s\right)\right] \\
\cdot Q^{\prime} g\left(x+v_{1} s, v_{1}, s\right) d s,
\end{array}
$$

with

$$
H(x+v t, t)=\int_{0}^{t} \int_{\left|v_{2}\right| \leqq K_{1}} f\left(x+v \tau, v_{2}, \tau\right) d v_{2} d \tau \sup k
$$

and

(2.5) $Q^{\prime} g\left(x, v_{1}, s\right)=Q g\left(x, v_{1}, s\right)+\int_{\left|v_{2}\right| \leqq K_{1}} g\left(x, v_{1}, s\right) g\left(x, v_{2}, s\right) d v_{2} \sup k$.

We notice that $Q^{\prime} g \geqq 0$, if $g \geqq 0$ and that $f$ satisfies (2.3). But by (2.1) and (2.5), 


$$
\begin{aligned}
\left\|Q^{\prime} f-Q^{\prime} g\right\|_{\infty} & \leqq\|Q f-Q g\|_{\infty}+K_{2}\left(\|f\|_{\infty}+\|g\|_{\infty}\right)\|f-g\|_{\infty} \\
& \leqq 2 K_{2}\left(\|f\|_{\infty}+\|g\|_{\infty}\right)\|f-g\|_{\infty} .
\end{aligned}
$$

Moreover by (2.2) and (2.4) for every $t_{0}>0$, there is a $C_{t_{0}}$, such that

$$
0<\exp [-H(x+v t, t)+H(x+v s, s)]<C_{t_{0}}
$$

for $0 \leqq s \leqq t \leqq t_{0}$. It follows by (2.6) and (2.7) that the $L^{\infty}$-solution of (2.3) is unique. For small $t$ it is the limit of the following convergent iteration scheme

$$
\begin{aligned}
& g_{1}= 0, \\
& g_{j}\left(x+v_{1} t, v_{1}, t\right)= \exp \left[-H\left(x+v_{1} t, t\right)\right] \cdot f_{0}\left(x, v_{1}, t\right) \\
&+\int_{0}^{t} \exp \left[-H\left(x+v_{1} t, t\right)+H\left(x+v_{1} s, s\right)\right] \\
& \cdot Q^{\prime} g_{j-1}\left(x+v_{1} s, v_{1}, s\right) d s, \quad j=2,3, \ldots .
\end{aligned}
$$

But $g_{2} \geqq g_{1} \geqq 0$ and by induction $\left(g_{j}\right)_{1}^{\infty}$ is a nonnegative, monotonically increasing sequence. Hence $g=\lim _{j \rightarrow \infty} g_{j} \geqq 0$. As $f$ is the unique solution, it follows that $\mathrm{g}=f \geqq 0$ for small $t$, and so by a continuation argument for all $t>0$.

THEOREM 2.2. The $L^{\infty}$-solution $f$ of (1.6) satisfies for $\varphi=1, v,|v|^{2}$ the moment conservation

$$
\int_{\Omega} f(x, v, t) \varphi(v) d x d v=\int_{\Omega} f_{0}(x, v) \varphi(v) d x d v
$$

and generates a weak solution to the equation of macroscopic gas dynamics

$$
\frac{\partial}{\partial t} \int f(x, v, t) \varphi(v) d v+\sum_{j=1}^{3} \frac{\partial}{\partial x_{j}} \int f(x, v, t) v_{j} \varphi(v) d v=0 .
$$

Proof. Using (1.3)-(1.5) we can easily show that

$$
\int Q f(x, v, t) \varphi(v) d v=0
$$

and then using (1.6) that

$$
\int_{\Omega} f(x+v t, v, t) \varphi(v) d v d x=\int_{\Omega} f_{0}(x, v) \varphi(v) d x d v .
$$

Hence the moment conservation holds. 
Multiplying (1.6) by $\varphi$ and a continuously differentiable function $\psi(x+v t, t)$ with period $\omega$ in $x$, and then integrating and applying (2.8) we get

$$
\begin{aligned}
\int f(x, v, t) \varphi(v) \psi(x, t) d v d x= & \int f_{0}(x, v) \varphi(v) \psi(x, 0) d x d v \\
& +\int_{0}^{t} \int f(x, v, s) \varphi(v) \frac{\partial}{\partial s} \psi(x, s) d v d x d s \\
& +\sum_{j=1}^{3} \int_{0}^{t} \int f(x, v, s) \varphi(v) v_{j} \frac{\partial}{\partial x_{j}} \psi(x, s) d v d x d s .
\end{aligned}
$$

These are the weak equations of macroscopic gas dynamics satisfied by $f$.

THEOREM 2.3. The $\mathscr{H}$-function

$$
\mathscr{H}(t)=\int_{\Omega} f(x, v, t) \log f(x, v, t) d x d v
$$

is nonincreasing as a function of $t$.

Proof. Evidently the standard proof of this $\mathscr{H}$-theorem holds in a formal sense in the present case. This formal proof is a strict proof for $0 \leqq t \leqq t_{0}$, if

$$
f(x, v, t)>\varepsilon>0 \quad \text { for }(x, v) \in \Omega, 0 \leqq t \leqq t_{0} \quad \text { (cf., e.g., [1]). }
$$

By (2.2)-(2.4) the solution $f_{n}$ of (1.6) with initial value $f_{0}+n^{-1} e^{-v^{2}}$ satisfies (2.9) for any $t_{0}>0$. Hence

$$
\int_{\Omega} f_{n}(x, v, t) \log f_{n}(x, v, t) d x d v
$$

is nonincreasing as a function of $t$ for $0 \leqq t \leqq t_{0}$. But $\lim _{n \rightarrow \infty} f_{n}=f$ in $L^{\infty}$, uniformly in $t$ for $0 \leqq t \leqq t_{0}$. We conclude that

$$
\mathscr{H}(t)=\int f(x, v, t) \log f(x, v, t) d x d v
$$

is nonincreasing as a function of $t$ for $t>0$.

\section{REFERENCE}

1. L. Arkeryd, On the Boltzmann equation. I. Existence, Arch. Rational Mech. Anal. (to appear).

Department of Mathematics, University of Goteborg, Fack S40220, Goteborg, SWEDEN

Current address: Department of Mathematics, Middle East Technical University, ANKARA, TURKEY 